\title{
PRE-HOSPITAL CARE
}

\section{Blue calls - time for a change?}

\author{
R Brown, J Warwick
}

\begin{abstract}
Prior alert via a landline telephone ("blue call") is commonly used to warn accident and emergency $(\mathrm{A} \& \mathrm{E})$ departments of the impending arrival of a seriously ill or injured patient. There are no published indications for making such calls or validated protocols on message content. Submitted telephone information has the potential for distortion as it is passed through the control centre resulting in inappropriate resource allocation. This study focuses on the quality and content of the message in the context of the available patient details as well as reviewing the clinical indications for the call. Data were collected on patients for whom "blue calls" were made to an A\&E department over three months of 1998. Patients with life threatening conditions who were brought by non-blue light ambulance were identified during the same period. Similar details were collected on these critical patients. Of the 189 "blue calls" with complete details, $73 \%$ were admitted, $(12 \%$ to ITU) and $18 \%$ died. Sixty nine per cent of cases were medical, $26 \%$ trauma and $5 \%$ obstetric. Pre-hospital observations were missing for $25 \%$ of patients (excluding patients in cardiac arrest), suggesting that the decisions to make a pre-alert call may have been based on subjective criteria in a significant minority. Information given over the telephone invariably included age, sex and presenting complaint but details of the current condition of the patient were included in only $11 \%$. On reviewing pre-hospital information, a consultant in $A \& E$ and an ambulance paramedic judged that a prior alert was justified in $93 \%$ but additional information would be helpful in $52 \%$ of cases to correctly mobilise resources. Seventy five "clinically critical" patients were found in the three months of the study. Clinically critical patients were patients who had no prior alert, transported by ambulance, who were subsequently admitted to intensive care, theatre, or other high dependency areas. They included 27 patients with symptoms of a myocardial infarction. These patients may have benefited from prior alert. A protocol is suggested to provide criteria for making a prior alert to the $A \& E$ department via a landline con-
\end{abstract}

nection. A standardised message structure would be used using vital signs and mechanism of injury or type of illness to assist in hospital preparation.

(Emerg Med f 2001;18:289-292)

Keywords: ambulance service

It is common practice in the United Kingdom for incoming ambulance crews to alert accident and emergency (A\&E) departments of the impending arrival of a seriously ill or injured patient. This allows allocation of resources, preparation of equipment and appropriate specialist teams to be assembled. The most common method of alert is a radio message to ambulance control who then telephone the hospital (personal communication). For many ambulance services, there are no agreed indications for prior alert or for message format or content. Inaccurate messages and inappropriate calls may cause poor allocation of resources, result in time wasted and the "cry wolf" effect. This study is based on prior alert calls received in a busy teaching hospital over a three month period, and surveys the content and accuracy of the message as well as the appropriateness of the call. The aims of the study were to identify whether the current method of prior alert in the London Ambulance Service (LAS) results in inappropriate deployment of hospital staff and if so whether this occurs from inaccurate message relay or incomplete information. In addition, possible undertriage may be identified by close examination of the "clinically critical" group (CC) of patients.

\section{Method}

The study took place at King's College Hospital $(\mathrm{KCH})$ South London, in conjunction with the LAS. Over a three month period from May to July 1998, patients were identified from a manual $\log$ of "blue calls" generated in the $\mathrm{A} \& \mathrm{E}$ department. This log records the exact message content as received by the A\&E staff. The date and time on this log was used to identify patients on the departmental computer system (Footman Walker) and in the LAS archives.

Documents available from the ambulance service included: crew documentation of events (patient report form, PRF), the central ambulance control (CAC) record of the call 
Table 1 Presenting complaints within "blue call" and critical cases groups

\begin{tabular}{lll}
\hline Presenting complaint & $\%$ of "blue calls" & $\%$ of critical cases \\
\hline Cardiac arrest & 20 & 0 \\
Chest pain & 5 & 55 \\
Difficulty in breathing & 18 & 8 \\
Fits & 8 & 2 \\
Haemorrhage & 1 & 2 \\
Obstetric/gynaecology & 6 & 6 \\
Overdose/poisoning & 3 & 2 \\
Trauma & 28 & 2 \\
Unconscious/collapse & 11 & 23 \\
\hline
\end{tabular}

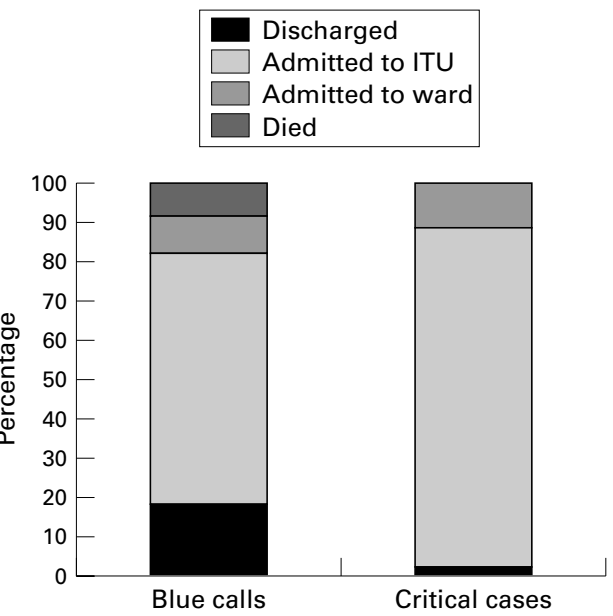

Figure 1 Outcomes for "blue calls" and critical cases shown as percentage of total.

(AS1), and tapes of communications between crew, CAC and hospital. Hospital documentation included the "blue call" $\log$, A\&E cards and the hospital notes for the patients identified.

For the CC group, comparative data were collected from the same sources (there was no prior alert message). CC patients were defined as: brought by non-blue light ambulance, and subsequently admitted directly to intensive care, coronary care, high dependency units, theatre, or who died in A\&E. These patients were identified by analysis of the Footman Walker database in A\&E.

Data collected for all patients included age, sex and vital signs recorded at scene. Present-

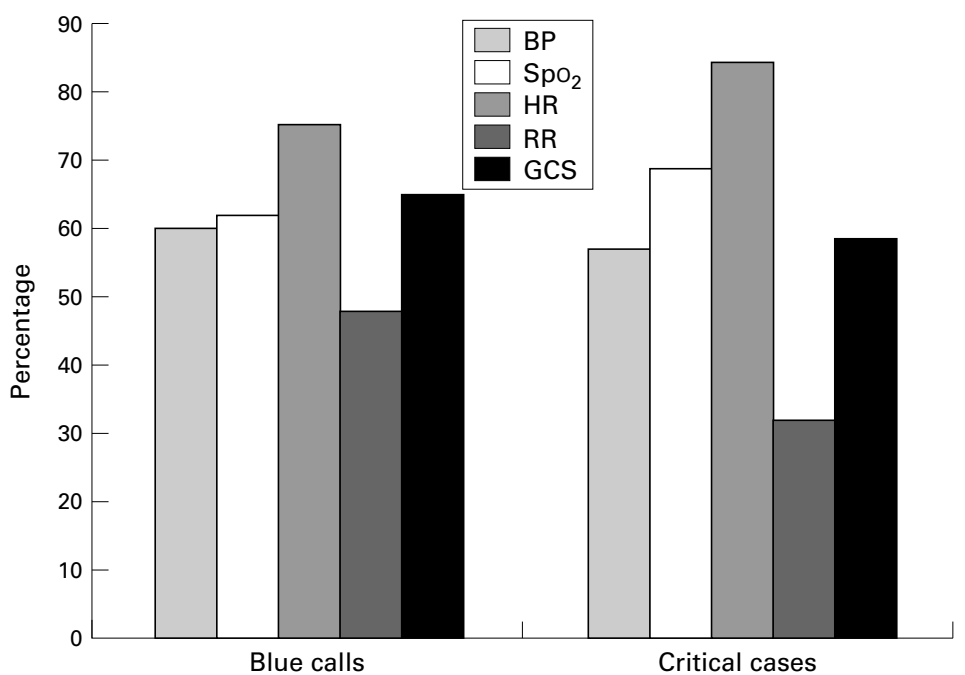

Figure 2 Percentages of patients who had vital signs recorded. ing complaints were categorised according to the LAS coding system into symptomatic or disease based categories. All data were collected manually and then entered in a Microsoft Access database for analysis. Patients were excluded for whom there was no PRF or hospital record available, or who arrived by Helicopter Emergency Service. Messages were reviewed in the light of the clinical information contained in the PRF alone. Both authors considered each case independently to decide whether the content of the message was reasonable in the light of the pre-hospital information. The authors also noted whether the prior alert was useful, and whether additional information would have assisted the hospital preparations. Criteria for useful information included whether the information would: activate the local trauma team, influence other specialist staff alert, or the preparation of specific equipment or drugs. The CC cases were reviewed on the basis of the PRF information. Judgement was made whether these patients could be identified as CC in the pre-hospital phase and whether a prior alert to the A\&E department may have been beneficial to their care.

\section{Results}

Two hundred and five "blue calls" were identified, of whom 189 (92\%) had complete details available. Sixty three per cent of these were men, the average age was 46 years with a range of 0-106 years. In the clinically critical group, $60 \%$ were men with an age range of 10-93 years and an average age of 58 years. Ambulance personnel recorded the blue call cases as trauma in $26 \%, 69 \%$ medical and obstetric in 5\%. Critical cases were $83 \%$ medical, $5 \%$ trauma and $12 \%$ obstetric. There was a bigger proportion of men in the blue call trauma category than for the medical category. The breakdown of presenting complaints for both the "blue calls" and critical cases are seen in table 1 .

The outcomes for "blue calls" and critical cases are seen in figure 1. There was one death in the critical cases group and a significant number of critical cases were admitted to intensive care during this period. There was one child in the CC group, for whom notes were not found, but who was admitted to an HDU bed for asthma.

Vital signs were recorded with variable consistency (figure 2). Cases of trauma tended to have vital signs recorded most frequently. Twenty five per cent of the "blue call" patients did not have any vital signs recorded. In this "blue call" group, $45 \%$ of patients with head injuries did not have a Glasgow coma score recorded, and $39 \%$ of patients labelled as difficulty in breathing did not have a respiratory rate recorded. The critical cases had at least one vital sign recorded in $96 \%$ of cases, and in $46 \%$ of these, there was at least one abnormal vital sign. Patients with chest pain in the CC group had abnormal vital signs recorded in $26 \%$ of cases compared with $50 \%$ of the patients with chest pain who had a prior alert made. In $55 \%$ of CC cases with chest pain, the 


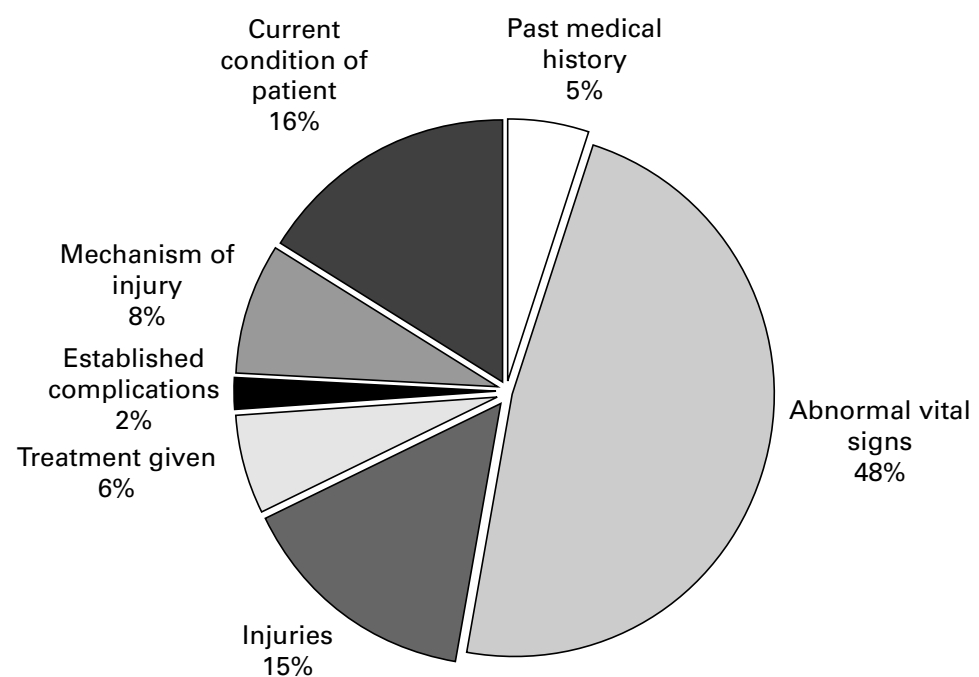

Figure 3 Type of missing information as percentage of total missing information for "blue calls".
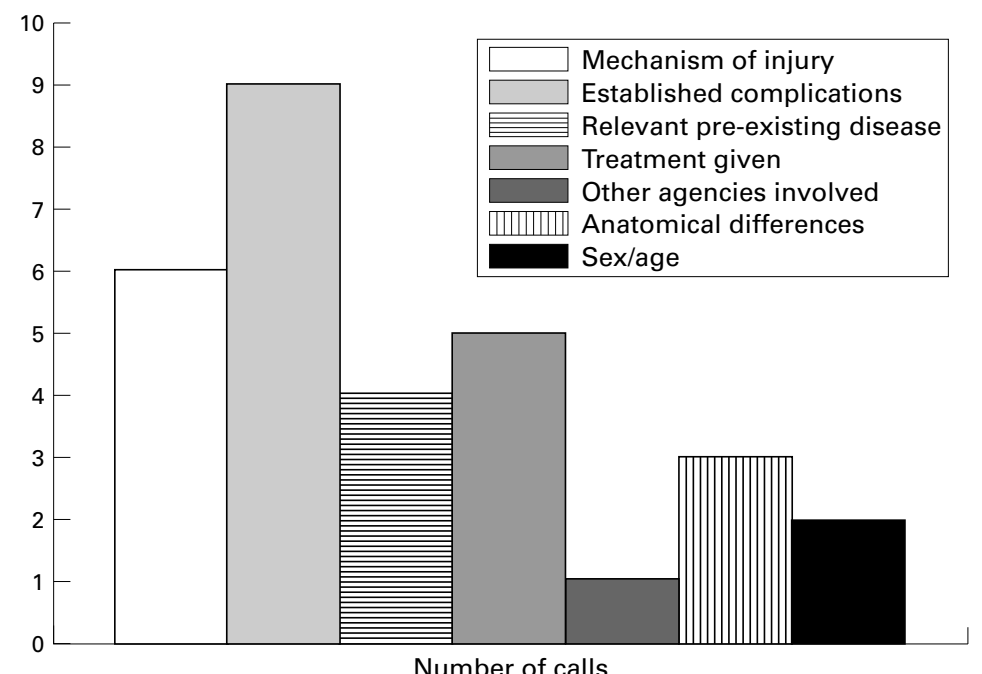

Figure 4 Categories of message differences.

symptoms recorded on the PRF were strongly suggestive of myocardial infarction compared with the "blue calls" where only $27 \%$ of the patients with chest pain had symptoms strongly suggestive of infarction.

Messages contained the age and sex of the patient in $99 \%$ of cases. Altogether 176 (93\%) of "blue calls" were considered to be appropriate given the clinical condition at scene. Seventy one per cent of messages contained useful information although in $52 \%$ of cases it is thought that further information would have been helpful. This "missing" information was available on the PRF documentation, but not passed on in the message. Figure 3 shows the types of missing information that would have contributed to decisions about resource allocation.

The message was changed by CAC in 30 $(16 \%)$ cases (fig 4$)$. This difference was not thought to have resulted in different action prior to the patient arriving at hospital. In addition there were nine instances when the recorded message in the $\mathrm{A} \& \mathrm{E} \log$ differed to the verbal message recorded on the tape $(A \& E$ reception error). Although the average estimated arrival time given by crews was the same as the average actual time to arrival, there was a significant discrepancy of over five minutes between estimated and arrival times for individual calls. There were four patients who arrived as the blue call was being taken.

In $42,(55 \%)$ of the CC patients, it was considered there was sufficient evidence on the PRF to indicate that the patient would benefit from a prior alert. Many (27) of these patients had chest pain with symptoms or signs suggestive of myocardial infarction. However, of the other 48 CC patients, $44 \%$ had abnormal vital signs recorded in the field or a mechanism of injury or illness type that would theoretically benefit from immediate medical care on arrival at hospital. Six per cent of these patients were admitted to ITU.

\section{Discussion}

The LAS receives over 650000 calls a year with no formal monitoring of blue calls. In 1998, KCH saw over 85000 patients, of which 21000 were brought by ambulance. Of the ambulance patients $4 \%$ were blue calls. $\mathrm{KCH}$ serves an inner city population that has a high incidence of serious violent crime. The proportion of trauma in this study is higher than previous studies (unpublished report and Wilson and Bleetman $\left.{ }^{1}\right)$. There is a relatively low incidence of myocardial infarction in the local population and this is reflected in the low numbers of cardiac arrest. The over representation of medical presentations in the critical cases group suggests that there is a lower threshold for making a prior alert in trauma than for medical conditions. There are no clear guidelines in the literature for field triage except for trauma ${ }^{2}$ and this may also influence the case mix within the blue call group. The LAS do not make clear recommendations for the indications for a blue call. ${ }^{3}$ Of 28 ambulance services questioned, three have protocols for trauma and two other services had protocol ${ }^{9}$ for specific hospitals (personal communication 1999). The actual criteria on which the crews based the decision to place a blue call are not clear. The high percentage of patients with no recorded vital signs suggests a lack of objective assessment at scene but is no different from LAS and other published figures. ${ }^{4-7}$ The evidence suggests that paramedics perform lifesaving interventions more often in the presence of abnormal vital signs. ${ }^{8}$ It may be that vital signs are being measured but not recorded because of the critical condition of the patient. Conversely, the high proportion of CC patients who had documented abnormal vital signs suggests under triage is occurring in these patients who may have benefited from immediate care on arrival at hospital.

Ambulance personnel can discriminate and identify specific conditions after additional training. ${ }^{9-12}$ The Manchester triage system reliably identifies critically ill and injured patients, ${ }^{13}$ but requires extensive training and takes significant time to apply. A simple protocol based on mechanism of injury and abnormal vital signs as discriminators would 
have prompted a prior alert for $55 \%$ of the CC group thus potentially improving patient care.

The authors judged the appropriateness of the prior alert on the documentation made by crews, reproducing the evidence available to crews as nearly as possible. Crew judgement relies on intuition ${ }^{14}$ and takes into account the scene and appearance of the patient. Even without these additional cues, most calls were thought by the authors to be appropriate. However, it was thought that more information should have been included in the message. The LAS recommend the message should include the hospital, sex of patient, and code letter indicating a category of presentation. ${ }^{3}$ This format, and particularly code letters, was not used once. Many ambulance services already use ASHICE (age, sex, history, injuries, condition, estimated time of arrival). Many of the message omissions in this study were a failure to pass on documented abnormal vital signs, or administered treatment and response as well as specific mechanism of injury. These items of information could be expected to change the actions at hospital. In many hospitals, the trauma team call out is based on mechanism of injury and abnormal vital signs. Additional equipment or drugs may be requested prior to the patient arriving if suitable information is given.

The travel time of 5.5 minutes is shorter than other studies, ${ }^{15}$ but in individual cases, variance from estimated time may lead to unnecessary deployment of hospital staff, and considerable delay for other patients. Conversely arrival before expected means ineffective preparation for the patients arrival.

There are anecdotal suggestions that relaying a message through a clerical worker at the hospital causes serious distortion and that messages should be received by medical personnel. Evidence from the literature suggests that physician on line advice reduces hospital stay and costs as well as improving decision making. ${ }^{16}$ The fact that there were no critical changes in the message content through five relays in this study suggests there is no major concern. Although six ambulance services in the UK reportedly have the capability to talk to a doctor by radio, in practice few use it (personal communication 1999).

Within the CC patients, because of the selection criteria for these patients, there were 27 patients with chest pain. The expected National Service Framework calls for a call to needle time of 60 minutes for myocardial infarction. A recent study showed transfer at emergency speed with sirens raises heart rate and cortisol concentrations in patients. ${ }^{17}$ Instead of transport at speed with blue lights, a prior alert may achieve short times by ensuring a physician and nurse are ready to receive the patient.
In conclusion, the current system for prior alert of hospitals seems to result in little over triage of patients, although there remains significant under triage of patients who would benefit from a prior alert. The message is consistently transmitted with little significant distortion despite multiple relays. The message lacks structure and content and relevant information is not passed on in a significant proportion of cases. A protocol for prior alert should be devised, using criteria based on the evidence in the literature, and including validated scores such as the Revised Trauma Score. It is possible that such a protocol would result in more "blue calls" being placed and hence over triage. However, a standardised message that contained vital signs if abnormal and details of mechanism of injury of illness would allow the hospital to grade their response appropriately. In addition, earlier physician intervention for the previously unidentified CC patients would be expected to improve care.

Contributors

Ruth Brown initiated the study, designed the protocol and collected and analysed hospital data and contributed to the discussed core ideas, John Warte the writing of the paper. Mr M Clayton, (paramedic) assisted in the writing of the paper. Mr M Clayton, (paramedic)
design of the database. Ruth Brown acts as guarantor.

Funding: none.

Conflicts of interest: none.

1 Wilson SNJ, Bleetman A. Emergency ambulance telephone lerts to an inner city accident and emergency departmen Prehospital Immediate Care 1999;3:154-5.

2 Maslanka AM. Scoring systems and triage from the field. Emerg Med Clin North Am 1993;11:15-27.

3 London Ambulance Service Standing order AO/44.

4 London Ambulance Service Asthma Audit. 1995 London Ambulance Service Audit Department.

5 London Ambulance Service Audit of Major Trauma 1997. London Ambulance Service Audit Department.

6 Moss RL. Vital signs records omissions on prehospital Moss RL. Vital signs records omissions on prehospital
patient encounter forms. Prehospital Disaster Medicine patient enco $1993 ; 8: 21-7$.

7 Gausche M, Henderson DP, Seidel JS. Vital signs as part of the prehospital assessment of the paediatric patient: a survey of paramedics. Ann Emerg Med 1990;19:173-9.

8 Brown LH, Prasad NH. Effect of vital signs of advanced life support interventions for prehospital patients. Prehospital Emergency Care 1997;1:145-8.

9 Harbison J, Massey A, Barnett L, et al. Rapid Ambulance protocol for acute stroke. Lancet 1999;353:1935.

10 Fries GR, McCalla G, Levitt MA, et al. A prospective comparison of paramedic judgement and the trauma triage rule in the prehospital setting. Ann Emerg Med 1994;24:885-9.

11 Banerjee S, Rhoden WE. Fast-tracking of myocardial infarctions by paramedics $\mathcal{f} R$ Coll Physicians Lond 1998;32:36-8.

12 Quinn T, Allan TF, Thompson DR, et al. Identification of patients suitable for direct admission to a coronary care unit by ambulance paramedics: an observational study. Prehospital Immediate Care 1999;3:126-30.

13 Cooke MW, Jinks S. Does the Manchester triage system detect the critically ill? F Accid Emerg Med 1999;16:179-81.

4 Benner P, Tanner C. Clinical judgement: how expert nurses use intuition. Am f Nurs 1987;87:23-31.

15 Harrison JF, Cooke MW. Study of early warning of accident and emergency departments by ambulance services. F Accid Emerg Med 1999;16:339-41.

16 Gerndt SJ, Conley JL, Lowell MJ, et al. Prehospital classification combined with an in-hospital trauma radio system response reduces cost and duration of evaluation of the injured patient. Surgery 1995;789-96.

17 Witzel K. Effects of emergency ambulance transportation on heart rate, blood pressure, corticotrophin and cortisol. Ann Emerg Med 1999;33:598-9. 